CCNY-HEP-04/10

November 2004

\title{
Kac-Moody theories for colored phase space (quantum Hall) droplets
}

\author{
Alexios P. Polychronakos \\ Physics Department, City College of the CUNY \\ Convent Avenue and $138^{\text {th }}$ Street, New York, NY 10031 \\ alexios@sci.ccny.cuny.edu
}

\begin{abstract}
We derive the canonical structure and hamiltonian for arbitrary deformations of a higher-dimensional (quantum Hall) droplet of fermions with spin or color on a general phase space manifold. Gauge fields are introduced via a Kaluza-Klein construction on the phase space. The emerging theory is a nonlinear higher-dimensional generalization of the gauged Kac-Moody algebra. To leading order in $\hbar$ this reproduces the edge state chiral Wess-ZuminoWitten action of the droplet.
\end{abstract}




\section{Introduction}

Describing fermions in terms of bosonic variables has been the source of much of our progress in understanding their many-body dynamics. Such descriptions are collectively termed bosonization [1]-[5].

An intuitive approach to such a description is to consider a dense collection of fermions forming a 'droplet' on their phase space and study the dynamics of the Fermi surface of the droplet. In two dimensions this leads to a chiral theory in $1+1$ dimensions [5].

In a previous paper [6] we used a phase space canonical approach to derive the Poisson structure and hamiltonian for arbitrary deformations of constant-density droplets for spinless (abelian) fermions on a general higher-dimensional phase space. This provided a nonlinear generalization of the results of Karabali and Nair [7] who derived a chiral boundary action for the higher-dimensional quantum Hall effect proposed by Zhang and $\mathrm{Hu}[\underline{8}$. The nonlinear terms derived in [6] captured higher order quantum corrections in the $1 / N$ approximation.

The analysis in [7] uses the quantum density matrix formulation and large-N approximations [9] and also applies to a nonabelian situation [10], where it leads to a generalization of the chiral sigma model known as the Wess-Zumino-Witten model 3]. This model describes boundary perturbations of the quantum Hall (droplet) state of fermions with color degrees of freedom.

The inclusion of spin, color or other internal degrees of freedom for the fermions in the phase space formulation presents new challenges, since the usual semiclassical droplet picture has to be modified and extended to accommodate the new degrees of freedom. Nevertheless, such a description is possible and leads to an interesting gauge generalization of the results for scalar particles. This will be derived in the present paper.

The organization of the paper is as follows. In section $\mathbf{2}$ we give a general analysis of phase space density dynamics and review the relevant results of [6]. In section 3 we introduce internal degrees of freedom as classical phase space variables and derive the corresponding droplet dynamics; we argue that the internal space needs to be quantized for an accurate description of the system and present the corresponding dynamics in terms of matrix generalizations of the boundary field obeying a generalized Kac-Moody algebra. We further demonstrate that the dynamics to leading order in $\hbar$ reproduce the Wess-Zumino-Witten model. In section 4 we introduce gauge degrees of freedom and define gauge transformations in the phase space 
structure, through a mechanism analogous to a quantum Kaluza-Klein reduction; we generalize the results of the previous section for the gauged case, reproducing a gauge Kac-Moody algebra and a gauged Wess-Zumino-Witten model. Finally, in the last section we discuss some outstanding issues.

Note on $\hbar$ : It is customary to put $\hbar=1$ and eliminate it from all expressions. In our case, however, we have to keep track of various orders of $\hbar$ in our calculations in order to reproduce the correct leading-order model. We could have kept the convention $\hbar=1$ and re-introduce it where appropriate and needed. For the sake of clarity, however, we preferred to keep $\hbar$ explicit as a book-keeping device, in order to indicate the appropriate scale of each term in the formulae, accepting the price of some $\hbar$-litter in the expressions.

\section{Review of phase space droplet dynamics}

\subsection{General formulation}

We shall start by considering noninteracting (spinless) particles on a general $D$ dimensional phase space manifold with coordinates $\phi^{\mu}, \mu=1, \ldots D$ and Poisson structure

$$
\left\{\phi^{\mu}, \phi^{\mu}\right\}_{s p}=\theta^{\mu \nu}
$$

where the subscript $s p$ stands for single-particle. For a non-degenerate Poisson structure the dimension $D$ should be even. The volume element in this phase space is

$$
d^{D} \phi=\frac{d \phi}{\sqrt{\theta}}, \text { where } d \phi=\prod_{\mu=1}^{D} d \phi^{\mu}, \quad \theta=\operatorname{det} \theta^{\mu \nu}
$$

The particles have hamiltonian $V(\phi)$ and perform classical motion:

$$
\dot{\phi}^{\mu}=\left\{\phi^{\mu}, V\right\}_{s p}=\theta^{\mu \nu} \partial_{\nu} V
$$

A dense collection of particles on this phase space is described in terms of its density $\rho(\phi, t)$. (This is essentially a phase space fluid dynamical description; for a recent review see [11.) The motion of the underlying particles induces a time dependence for the density $\rho$. Its time evolution is given by a canonical transformation generated by $V$ :

$$
\dot{\rho}=\{\rho, V\}_{s p}=\theta^{\alpha \beta} \partial_{\alpha} \rho \partial_{\beta} V
$$


We can obtain the same dynamics without referring to the underlying particles by assuming a hamiltonian and canonical structure for the field $\rho$

$$
\dot{\rho}=\{\rho, H\}
$$

[These brackets should not be confused with the single-particle brackets (11).] Choosing as hamiltonian the total particle energy

$$
H=\int \frac{d \phi}{\sqrt{\theta}} \rho V
$$

the appropriate Poisson brackets are

$$
\left\{\rho\left(\phi_{1}\right), \rho\left(\phi_{2}\right)\right\}=\sqrt{\theta\left(\phi_{+}\right)} \theta^{\mu \nu}\left(\phi_{+}\right) \partial_{\mu} \rho\left(\phi_{+}\right) \partial_{\nu} \delta\left(\phi_{-}\right)
$$

where we defined relative and mid-point coordinates $\phi_{-}=\phi_{1}-\phi_{2}$ and $\phi_{+}=\frac{\phi_{1}+\phi_{2}}{2}$.

The above brackets (17) are the standard infinite-dimensional Poisson algebra of functions on the phase space manifold. In terms of test functions their form becomes more obvious: defining

$$
\rho[F]=\int \frac{d \phi}{\sqrt{\theta}} F(\phi) \rho(\phi)
$$

for some function on the phase space $F$, then the brackets of two such integrals are

$$
\{\rho[F], \rho[G]\}=\rho\left[\{F, G\}_{s p}\right]
$$

In deriving the above we used the identity

$$
\partial_{\mu}\left(\frac{\theta^{\mu \nu}}{\sqrt{\theta}}\right)=0
$$

which is a corollary of the Bianchi identity for $\theta^{\mu \nu}$. The equation of motion (4) arises as the canonical evolution with hamiltonian (6).

The above algebra has Casimirs. For any function of a single variable $f(x)$, the integral

$$
C[f]=\int \frac{d \phi}{\sqrt{\theta}} f(\rho)
$$

has vanishing Poisson brackets with $\rho$ and constitutes a Casimir. There are, thus, an infinite tower of Casimirs spanned by $C_{n} \equiv C\left[x^{n+1}\right]$ for $n=0,1,2, \ldots$. 


\subsection{Droplet dynamics}

We now specialize to the case where the particles underlying the density $\rho$ are (temporarily spinless) fermions. A dense collection of fermions in phase space will form a Fermi liquid. Semiclassically, the fermions will fill densely a region of the phase space, with one particle per volume $(2 \pi \hbar)^{D / 2}$, forming a constant density droplet of arbitrary shape. Under time evolution, the density remains constant inside the droplet (by Liouville's theorem) while each point on its boundary moves according to the single-particle equation of motion, thus deforming the shape of the droplet.

To describe the droplet it suffices to determine the shape of its $D$-1-dimensional boundary. This can be done by expressing one of the phase space coordinates on the boundary, say $\phi^{D} \equiv R$, as a function of the remaining phase space coordinates $\sigma^{\alpha}$. (In the sequel we use early greek letters for the set of indices $\alpha=1,2, \ldots D-1$ while middle greek letters will take values in the full $D$-dimensional space.) So the dynamical variable is the function $R(\sigma, t)$. (For a finite droplet, it is convenient to assume that the origin of coordinates is inside the droplet and to think of $R$ as a 'radial' coordinate and $\sigma^{i}$ as 'angular' coordinates.)

The canonical structure of the droplet variable $R$ arises from a hamiltonian reduction of the full density canonical structure: a constant-density droplet of arbitrary shape constitutes a particular class of density functions and thus a submanifold of the full manifold of configurations for $\rho$, of the form

$$
\rho=\rho_{o} \vartheta\left(R-\phi^{D}\right)
$$

where $\vartheta(x)=\frac{1}{2}[1+\operatorname{sgn}(\mathrm{x})]$ is the step function. To find the droplet Poisson brackets we need to project the canonical two-form of $\rho$ on this submanifold. This can be done with the help of the so-called cartographic transformation of the density $\rho$. (We refer the reader to [6] for further details and a full derivation). Introducing the shorthand $f_{b}$ for quantities defined on the boundary of the droplet,

$$
f_{b} \equiv f\left(\phi^{D}=R, \sigma^{\alpha}\right)
$$

the induced Poisson brackets for $R$ are expressed as

$$
\left\{R\left(\sigma_{1}\right), R\left(\sigma_{2}\right)\right\}=\frac{\sqrt{\theta_{b+}}}{\rho_{o}}\left[\theta_{b+}^{D \alpha} \partial_{\alpha} \delta\left(\sigma_{-}\right)-\theta_{b+}^{\alpha \beta} \partial_{\alpha} R_{+} \partial_{\beta} \delta\left(\sigma_{-}\right)\right]
$$

and the hamiltonian becomes

$$
H=\rho_{o} \int \frac{d \phi}{\sqrt{\theta}} V(\phi) \vartheta\left(R-\phi^{D}\right)
$$


As before, + and - stand for mid-point and relative $\sigma$ coordinates.

The above Poisson structure and hamiltonian encode the full dynamics of the droplet and imply the canonical evolution for the boundary

$$
\dot{R}=\theta_{b}^{D \alpha} \partial_{\alpha} V_{b}-\theta_{b}^{\alpha \beta} \partial_{\alpha} R \partial_{\beta} V_{b}
$$

This equation refers only to the boundary, although the hamiltonian is defined in the bulk of the droplet. The same equation can be obtained by following the singleparticle evolution of the particles on the boundary of the droplet 6 .

It is worth noting that if the coordinate $\phi^{D}$ is chosen to parametrize the potential (that is, surfaces $\phi^{D}=$ constant are equipotential), $V=V\left(\phi^{D}\right)$, the second term above drops and we get

$$
\dot{R}=\theta_{b}^{D \alpha} \partial_{\alpha} V_{b}
$$

In the special case when $\theta_{b}^{D \alpha}$ is nonzero only for a single value of the index $\alpha$ (there is a global variable conjugate to $\phi^{D}$, call it $\phi^{1}$ ), and is a function only of $\phi^{D}$, the above equation becomes

$$
\dot{R}=\theta_{b}^{01} V^{\prime}{ }_{b} \partial_{1} R
$$

which can easily be solved by a hodographic transformation, interchanging $R$ and $\phi^{1}$.

We should warn that the droplet may have more than one boundaries, depending on its topology. In such cases we need to introduce several commuting boundary fields $R_{n}$, one for each boundary. Similarly, the boundary could intersect $\sigma=$ constant lines at more than one $\phi^{D}$, in which case we need again to introduce several boundary fields, one for each branch, with appropriate matching conditions tying them into a unique boundary.

We conclude this section with the following remarks:

1. The Poisson brackets (14) of $R$ contain an affine 'chiral' part (the first term in the bracket) as well as an ordinary Poisson density structure over the gauge manifold $\left\{\sigma^{\alpha}\right\} / \phi^{D}$. The quotient arises because $\theta^{\alpha \beta}$ is degenerate, being odd-dimensional, and effectively the variable conjugate to $\phi^{D}$ drops out.

2. (14) satisfies the Bianchi identity, as a corollary of the Bianchi identity of the Poisson brackets for $\rho$, although its direct check is highly nontrivial. In the special case when $\theta^{\mu \nu}$ is independent of $\phi^{D}$ the affine and linear terms decouple and individually satisfy the Bianchi identity. In the generic case, however, both terms are needed to satisfy the identity. This will be relevant to the case of particles with internal degrees of freedom. 
3. The Casimirs of the original density for the droplet become $C_{n}=C_{0}$. So they are all neutralized, the only remaining Casimir being the total particle number $C_{0}=N$.

4. The constant $\rho_{o}$ appears both in the hamiltonian and the Poisson brackets and is irrelevant for classical dynamics. The semiclassical interpretation of the droplet, however, fixes the value $\rho_{o}=1 /(2 \pi \hbar)^{D / 2}$, which will be important for quantization and for the case of spinning particles.

\section{Particles with internal degrees of freedom}

The generalization of the above semiclassical construction for fermions with internal degrees of freedom (spin, color, flavor etc.) presents some conceptual problems, due to the fact that internal quantum numbers are never really classical.

One approach would be to view the internal degrees of freedom simply as different species of fermions and apply the above procedure separately for each species. We would obtain a set of partially overlapping droplets with mutually commuting boundary fields $R$. This, however, has several drawbacks. One is that such a description would not allow the particle number of each species to fluctuate (remember that the total particle number for each droplet is a Casimir), thus excluding the possibility of having transitions of fermions from one species to the other. Another related problem is that the hamiltonian may not be diagonal in the particular chosen basis of flavors. This would happen, e.g., for a spin-dependent hamiltonian or in the case where we include gauge fields that act on the spins or colors.

We need, therefore, to start from a proper semiclassical description of the full set of degrees of freedom without the above limitations. This will be done in this section.

\subsection{Introducing spins as classical phase space variables}

We shall start from a description where the classical phase space encodes also the internal degrees of freedom of the particles. This will be done by considering the internal quantum numbers as arising from the quantization of an internal, compact phase space for the particles. For shortness, we shall refer to this space as representing 'spin' variables, understanding that it can also represent color, flavor or any other internal degrees of freedom.

Consider the direct product of the original phase space $\theta^{\alpha \beta}$ and an additional 
compact phase space with coordinates $\xi^{i}$ and Poisson structure $\theta^{i j}$ (middle latin letters will stand for indices of the components of this compact phase space). Clearly $\theta^{\alpha i}=0$. The dimensionality $D_{I}$ of the $\xi$ space will be left arbitrary. The total volume of this space, however, will be chosen as $n(2 \pi \hbar)^{D_{I} / 2}$. We see that the size of this space is microscopic, involving Planck's constant.

Semiclassically, we will have one quantum state per volume $(2 \pi \hbar)^{D_{I} / 2}$ in the internal phase space. The above choice of volume implies that we shall have $n$ quantum states associated with this space, thus endowing the particles with $n$ internal states. The classical variables $\xi^{i}$ represent spin operators for the particle. The droplet procedure of the previous section can, then, be applied to the total phase space $(\phi, \xi)$.

We shall choose conventions in which the canonical two-form of the spin space $\omega_{i j}$ is of order $\hbar$ while the range of the coordinates $\xi^{i}$ is of order $\hbar^{0}$. This makes the Poisson structure $\theta^{i j}$ of order $1 / \hbar$. We shall also take the internal phase space to be homogeneous and the determinant of $\theta^{i j}$ equal to $(2 \pi \hbar)^{-D_{I}}$, therefore making the determinant of the total Poisson structure independent of $\xi^{i}$

$$
\operatorname{det}\left(\theta^{\mu \nu} \times \theta^{i j}\right)=\operatorname{det}\left(\theta^{\mu \nu}\right) \operatorname{det}\left(\theta^{i j}\right)=(2 \pi \hbar)^{-D_{I}} \theta
$$

There are many potential realizations of the spin space $\xi^{i}$. A specific example of such a space is $S^{2}$ with canonical two-form proportional to the area form. Choosing $\xi^{1}=\varphi / 2 \pi, \xi^{2}=(n / 2) \cos \theta$, with $(\theta, \varphi)$ polar and azimuthal angles on the sphere, the canonical two-form and Poisson structure will be

$$
\omega=\frac{\hbar n}{2} \sin \theta d \theta d \phi=2 \pi \hbar d \xi^{1} d \xi^{2} \rightarrow\left\{\xi^{2}, \xi^{1}\right\}=\frac{1}{2 \pi \hbar}
$$

The range of $\left(\xi_{1}, \xi_{2}\right)$ is $(1, n)$ and the total area of this space is $2 \pi \hbar n$. Semiclassically it can support $n$ quantum states. The quantization of this phase space reproduces the lowest Landau level of a particle on the sphere with a magnetic monopole of strength $n$ at the center. It is known that these states form a spin- $\frac{n}{2}$ multiplet of the group of rotations, the cartesian coordinates of the particle becoming spin- $\frac{n}{2} S U(2)$ matrices. The number of states is $2 j+1=n+1$, the shift due to the nonzero curvature of the space.

Another realization of the internal phase space would be the grassmanian manifold $G=U(M) / U\left(M_{1}\right) \times \cdots \times U\left(M_{k}\right)\left(M_{1}+\cdots+M_{k}=M\right)$ with an appropriate canonical form. This can be visualized as the lowest Landau level of a particle moving on the group manifold $U(M)$ with an appropriate magnetic field. The canonical 
structure $\omega=d \mathcal{A}$ is determined by the Kirillov one-form

$$
\mathcal{A}=i \hbar \operatorname{tr}\left(K U^{-1} d U\right)
$$

where $U$ is a $U(M)$ matrix and $K$ is a hermitian $M \times M$ matrix that can be chosen diagonal. The above is invariant under right-multiplication of $U$ by a unitary matrix commuting with $K$, so the corresponding $U(N)$ coordinates have to be eliminated. The phase space manifold, then, is the grassmanian $G$ where $M_{1}, \ldots M_{k}$ are the degeneracies of the eigenvalues of $K$.

Quantization of the above phase space requires that the eigenvalues of $K$ be integers (a condition akin to the monopole quantization on the sphere). The quantum states will form irreducible representations of $U(M)$ with lengths of Young tableau rows given by the eigenvalues of $K$ and $U(1)$ charge equal to the total number of boxes. So this phase space will reproduce internal 'color' quantum numbers in a given representation of $S U(M)$ and the classical coordinates $\xi^{i}$ represent color matrices in this representation. The previous $S^{2}$ monopole construction can be realized as the grassmanian manifold $U(2) / U(1) \times U(1)$ with the two eigenvalues of $K$ differing by $n$.

The exact realization of the spin space is unimportant at this point. The only thing that matters is the fact that we will have $n$ internal states for each fermion. Specific realizations, however, will be more convenient depending on the dynamics and symmetries of the problem, as will be apparent later.

\subsection{Realization of droplets with internal degrees of freedom}

We are now set to apply the droplet formalism to the problem. The total phase space has dimension $D+D_{I}$ and can accommodate one fermion per volume $(2 \pi \hbar)^{\left(D+D_{I}\right) / 2}$. Fermions on this space will form a droplet with density $\bar{\rho}_{o}=1 /(2 \pi \hbar)^{\left(D+D_{I}\right) / 2}$, reserving the notation $\rho_{o}=1 /(2 \pi \hbar)^{D / 2}$ for the density in coordinate phase space. The droplet boundary variable $R$ will be a function of both $\sigma^{\alpha}$ and $\xi^{i}$.

The Poisson brackets of $R$ are given by the general formula (14) applied to the present phase space structure:

$$
\begin{aligned}
& \left\{R\left(\sigma_{1}, \xi_{1}\right), R\left(\sigma_{2}, \xi_{2}\right)\right\}=\frac{\sqrt{\theta_{b+}}}{\rho_{o}} \quad\left[\theta_{b+}^{D \alpha} \partial_{\alpha} \delta\left(\sigma_{-}\right) \delta\left(\xi_{-}\right)-\theta_{b+}^{\alpha \beta} \partial_{\alpha} R_{+} \partial_{\beta} \delta\left(\sigma_{-}\right) \delta\left(\xi_{-}\right)\right. \\
& \left.-\theta_{+}^{i j} \partial_{i} R_{+} \partial_{j} \delta\left(\xi_{-}\right)\right]
\end{aligned}
$$

where we used (19) and $(2 \pi \hbar)^{D_{I} / 2} \bar{\rho}_{o}=\rho_{o}$. Assuming a particle hamiltonian $V(\phi, \xi)$ 
that depends also on the spin variables, the hamiltonian for $R$ will be

$$
H=\rho_{o} \int \frac{d \phi d \xi}{\sqrt{\theta}} V(\phi, \xi) \vartheta\left(R(\sigma, \xi)-\phi^{D}\right)
$$

The above would be an exact classical description of the droplet. The fact, however, that the internal space is of Planck size and supports a few quantum states renders it essentially quantum mechanical and makes the classical description inadequate. We need, therefore, to quantize the internal degrees of freedom and incorporate them in the Poisson brackets for $R$. This can be done by quantizing the spin coordinates $\xi$.

This quantization goes along standard lines. The $\xi^{i}$ become noncommutative and are represented by $n \times n$ matrices. Functions of the $\xi^{i}$ become matrices on the $n$-dimensional internal Hilbert space; real functions such as $R(\xi)$ become hermitian matrices $R^{a b}$, where early latin letters $a, b, \ldots=1, \ldots n$ will stand for spin indices. Integration over the phase space $\xi$ amounts to summing over Hilbert space states with a volume of $(2 \pi \hbar)^{D_{I} / 2}$ per state, that is, a trace over the Hilbert space

$$
\int \frac{d \xi}{\sqrt{\operatorname{det}\left(\theta^{i j}\right)}} \rightarrow(2 \pi \hbar)^{D_{I} / 2} \operatorname{tr} \quad \text { or } \quad \int d \xi \rightarrow \operatorname{tr}
$$

while the $\xi$-Poisson brackets become matrix commutators over $i \hbar$ :

$$
\{A, B\}_{\xi} \equiv \theta^{i j} \partial_{i} A \partial_{j} B \rightarrow \frac{1}{i \hbar}[A, B]
$$

We also need the Dirac delta-function $\delta\left(\xi_{1}-\xi_{2}\right)$. Since this relates two different points in the $\xi$ space it should carry two sets of matrix indices $\left(a_{1}, b_{1} ; a_{2}, b_{2}\right)$ and implement the defining property

$$
\int d \xi_{1} F\left(\xi_{1}\right) \delta\left(\xi_{1}-\xi_{2}\right)=F\left(\xi_{2}\right)
$$

This implies for matrix quantities

$$
\operatorname{tr}_{1}\left(F_{1} \delta_{12}\right)=F_{2}
$$

where the subscripts 1,2 refer to the first and second set of matrix indices. This means that $\delta_{12}$ is proportional to the matrix that exchanges the matrix spaces 1 and 2 , which is written in terms of Kronecker deltas as:

$$
\left(\delta_{12}\right)^{a_{1} b_{1} ; a_{2} b_{2}}=\delta^{a_{1} b_{2}} \delta^{a_{2} b_{1}}
$$

and satisfies

$$
F_{1} \delta_{12}=\delta_{12} F_{2}, \quad F_{2} \delta_{12}=\delta_{12} F_{1}
$$

Now we may determine the dynamics of the matrix field variable $R$. 


\subsubsection{Poisson structure}

In order to obtain Poisson brackets for the matrix variable $R$ in a form that is not too unwieldy we shall assume that $\theta^{\mu \nu}$ is independent of $\phi^{D}$. This can always be achieved with an appropriate choice of coordinates; full generality can be restored after obtaining the Poisson brackets of $R$ by performing the inverse change of variables. This choice guarantees that $\theta_{b}^{\mu \nu}$ and $\sqrt{\theta_{b}}$ become independent of $R$ and are scalar functions of $\sigma$.

To translate the expression in (22) to matrix spin variables, observe that the last term in the Poisson brackets of $R$, involving $\xi$-space derivatives, can be written as

$$
\theta_{+}^{i j} \partial_{i} R_{+} \partial_{j} \delta\left(\xi_{-}\right)=\left\{R\left(\xi_{1}\right), \delta\left(\xi_{1}-\xi_{2}\right)\right\}_{\xi_{1}}=-\left\{R\left(\xi_{2}\right), \delta\left(\xi_{1}-\xi_{2}\right)\right\}_{\xi_{2}}
$$

This translates to the matrix expression

$$
\frac{1}{i \hbar}\left[R_{1}, \delta_{12}\right]=-\frac{1}{i \hbar}\left[R_{2}, \delta_{12}\right]
$$

the equality of the two expressions being ensured by (29). We also have to express functions $F_{+}$defined on mid-point coordinates $\xi_{+}$in terms of matrix indices. For this, we remark that, when $F_{+}$multiplies delta functions or their derivatives, it can also be expressed as

$$
F_{+}=F\left(\frac{\xi_{1}+\xi_{2}}{2}\right)=\frac{F\left(\xi_{1}\right)+F\left(\xi_{2}\right)}{2}
$$

and this translates to $\frac{1}{2}\left(F_{1}+F_{2}\right)$ in matrix notation.

We now have all the ingredients. Each classical expression in (22) becomes a corresponding matrix in the spin space with a residual dependence on $\sigma^{\alpha}$; subscripts + and - refer to mid-point and relative $\sigma$ coordinates. Altogether we obtain

$$
\begin{aligned}
\left\{R^{a b}\left(\sigma_{1}\right), R^{c d}\left(\sigma_{2}\right)\right\}=\frac{\sqrt{\theta_{+}}}{\rho_{o}} & {\left[\theta_{+}^{D \alpha} \partial_{\alpha} \delta\left(\sigma_{-}\right) \delta^{a d} \delta^{c b}\right.} \\
& -\frac{1}{2} \theta_{+}^{\alpha \beta}\left(\partial_{\alpha} R_{+}^{a d} \delta^{c b}+\partial_{\alpha} R_{+}^{c b} \delta^{a d}\right) \partial_{\beta} \delta\left(\sigma_{-}\right) \\
& \left.-\frac{1}{i \hbar}\left(R_{+}^{a d} \delta^{c b}-R_{+}^{c b} \delta^{a d}\right) \delta\left(\sigma_{-}\right)\right]
\end{aligned}
$$

Note that the last term involves an explicit $\hbar$.

These brackets are a generalization of the Kac-Moody algebra. To make this explicit, define the generators $T^{A}, A=1,2, \ldots n^{2}-1$ of the fundamental representation of $S U(n)$ (capital latin letters will stand for $U(N)$ generator indices) and

append the $U(1)$ generator (proportional to the $n \times n$ unit matrix) $T^{0}=I / \sqrt{n}$ for 
the full set of generators of $U(n)$. We choose their normalization so that they satisfy the orthogonality and completeness conditions

$$
\operatorname{tr}\left(T^{A} T^{B}\right)=\delta^{A B}, \quad \sum_{A=0}^{n^{2}-1}\left(T^{A}\right)^{a b}\left(T^{A}\right)^{c d}=\delta^{a d} \delta^{c b}
$$

Their commutators define the $S U(N)$ structure constants

$$
\left[T^{A}, T^{B}\right]=i f^{A B C} T^{C}
$$

(with $f^{0 A B}=0$ ). We further define the symmetrized trace

$$
d^{A B C}=\frac{1}{2} \operatorname{tr}\left(T^{A} T^{B} T^{C}+T^{B} T^{A} T^{C}\right)
$$

The symbol $d^{A B C}$ is also known as the anomaly of the group $U(n)$, since it appears in the evaluation of the triangle anomaly graphs in $3+1$-dimensional gauge theories.

The matrices $T^{A}$ can be used as a basis to express the hermitian matrix $R$ :

$$
R^{a b}=\sum_{A} R^{A}\left(T^{A}\right)^{a b}, \quad R^{A}=\operatorname{tr}\left(R T^{A}\right)
$$

In terms of the dynamical variables $R^{A}$ the Poisson brackets (133) become

$$
\begin{aligned}
& \left\{R^{A}\left(\sigma_{1}\right), R^{B}\left(\sigma_{2}\right)\right\}=\frac{\sqrt{\theta_{+}}}{\rho_{o}} \quad\left[\theta_{+}^{D \alpha} \partial_{\alpha} \delta\left(\sigma_{-}\right) \delta^{A B}\right. \\
& \left.-d^{A B C} \theta_{+}^{\alpha \beta} \partial_{\alpha} R_{+}^{C} \partial_{\beta} \delta\left(\sigma_{-}\right)+\frac{1}{\hbar} f^{A B C} R_{+}^{C} \delta\left(\sigma_{-}\right)\right]
\end{aligned}
$$

We recognize this as a Kac-Moody type algebra generalized to higher dimension with an additional term proportional to the anomaly of $U(n)$.

This algebra inherits the Casimir $C_{0}$ of the classical algebra. The integral

$$
N=\rho_{o} \int \frac{d \sigma}{\sqrt{\theta}} \operatorname{tr} R=\rho_{o} \int \frac{d \sigma}{\sqrt{\theta}} \sqrt{n} R^{0}
$$

commutes with $R$. This is the total particle number.

\subsubsection{Hamiltonian}

The hamiltonian of the droplet can similarly be expressed in matrix form. The only question is matrix ordering. A $\xi$-dependent single-particle hamiltonian $V(\phi, \xi)$ and its product with the step function $\vartheta\left(R-\phi^{D}\right)$ of a matrix $R$ introduce ordering ambiguities. 
Ordering will not be an issue as long as $V$ is at most linear in the $\xi^{i}$. This is, in fact, related to the question of the specific realization of the internal spin space, as commented at the end of the previous section and as we shall analyze presently.

Remember that the spin space is microscopic (of order $\hbar$ ). The relevant variables are really $S^{i}=\hbar \xi^{i}$, representing quantum spin operators, and their products are higher order in $\hbar$. Such higher-order terms will be equally relevant only if they come with large coefficients (of order $1 / \hbar$ for quadratic terms etc.), which is unnatural. This, however, can be avoided if the internal phase space is chosen wisely so that all spin terms in the hamiltonian can be expressed linearly in the coordinates $\xi^{i}$. This can best be illustrated with the following example.

Consider the case that there are $n$ internal states. As exposed in the previous section, we may realize them either as a spin- $\frac{n-1}{2}$ representation of $S U(2)$, in terms of an $S^{2}$ phase space, or as the fundamental representation of $S U(n)$, in terms of the grassmanian space $U(n) / U(n-1) \times U(1)$, that is, the Kirillov action (21) with $K=\operatorname{diag}(1,0, \ldots, 0)$.

In either case the Hilbert space is $n$-dimensional, so there are $n^{2}$ linearly independent hermitian operators on this space (including the identity operator) that can be used to expand any operator. In the $S U(2)$ case these are the three spin matrices $S^{1,2,3}=\hbar \xi^{1,2,3}$ (the $\xi^{i}$ are Pauli or higher-spin $S U(2)$ matrices) as well as their ordered products up to degree $n-1$. A general spin-dependent term in the single-particle hamiltonian, then, can be expressed as a sum of such monomials. In the $S U(n)$ realization, however, the $n^{2}-1$ fundamental generators (represented by coordinates $\xi^{i}$ ) are a complete set and therefore any hamiltonian can be expressed as a linear expression in the $\xi^{i}$. Clearly, physics originating from $S U(2)$ spin will only involve linear expressions in the $S^{i}$, while physics originating from color or flavor degrees of freedom will give rise to a linear expression in the full set of $S U(n)$ generators.

In conclusion, an appropriate choice of realization of the internal states will lead to an expression for $V(\phi, \xi)$ linear in $\xi$ and we may write

$$
V(\phi, \chi)=\bar{V}(\phi)+V_{i}(\phi) \xi^{i} \equiv V_{0}+\hbar \hat{V}
$$

where $\bar{V}$ is the spinless part and $\hbar \hat{V}=V_{i} \xi^{i}$ is the spin part of the single-particle energy (where we explicitly indicate the fact that it is of order $\hbar$ ). The $V_{i}$ are 'magnetic field' terms. The matrix representation of $V$ is unambiguous. Further, there are no ordering problems in the definition of $\vartheta\left(R-\phi^{D}\right)$, since it is defined pointwise in $\sigma$ and does not couple matrices $R$ at different points that could be 
noncommuting. We may define it, for instance, via the expression

$$
\vartheta\left(R-\phi^{D}\right)=-\frac{1}{2 \pi i} \int \frac{d k}{k+i \varepsilon} e^{i k\left(R-\phi^{D}\right)}
$$

The multiplication of $V$ and $\vartheta\left(R-\phi^{D}\right)$ inside the integral is also free of ordering issues: $V$ is linear in $\xi$ and the trace representing $\xi$-integration makes its exact placement immaterial.

Altogether the droplet hamiltonian will be given by (23) with the integral expressed as a trace

$$
H=\rho_{o} \operatorname{tr} \int \frac{d \phi}{\sqrt{\theta}} V(\phi, \xi) \vartheta\left(R(\sigma, \xi)-\phi^{D}\right)
$$

The matrix expression inside the trace can also be obtained by integrating the classical expression in terms of $\phi^{D}$ and then promoting $R$ to a matrix, which will give the same result as using the matrix definition of $\vartheta\left(R-\phi^{D}\right)$.

The above Poisson structure and hamiltonian imply an equation of motion for $R: \dot{R}=\{R, H\}$. In evaluating this Poisson bracket it is useful to use the relations

$$
F_{+}=\frac{F\left(\sigma_{1}\right)+F\left(\sigma_{2}\right)}{2}, \quad F_{+} G_{+}=\frac{F\left(\sigma_{1}\right) G\left(\sigma_{2}\right)+F\left(\sigma_{2}\right) G\left(\sigma_{1}\right)}{2}
$$

which hold true for any two functions $F, G$ when multiplying the delta-function $\delta\left(\sigma_{-}\right)$or its derivatives. We obtain

$$
\dot{R}=\theta_{b}^{D \alpha} \partial_{\alpha} V_{b}-\frac{1}{2} \theta_{b}^{\alpha \beta}\left(\partial_{\alpha} R \partial_{\beta} V_{b}+\partial_{\beta} V_{b} \partial_{\alpha} R\right)-\frac{1}{i \hbar}\left[R, V_{b}\right]
$$

This is the matrix version of the equation of motion (16) with a symmetric ordering of the middle term.

\subsection{The leading-order action}

Assuming that the droplet deviates slightly from an equilibrium configuration, we can analyze its motion as a perturbation of its equilibrium shape. This will be useful for large droplets (large number of particles) where we can recover a boundary action to leading order in $\hbar$.

Consider a reference droplet configuration filling the phase space up to an energy level $E_{o}$ for the scalar part of the single-particle hamiltonian $V_{0}$. That is, the boundary field of the reference configuration $R=r_{o}(\sigma)$ is $\xi$-independent (proportional to the identity matrix) and satisfies

$$
V_{0}\left(r_{o}(\sigma), \sigma\right)=E_{o}=\text { constant }
$$


(From now on, a subscript ${ }_{o}$ in any quantity will signify the value of the quantity at $\phi^{D}=r_{o}$.)

Note that $r_{o}(\sigma)$ is not a time-independent solution of the equations of motion, since it does not minimize the full hamiltonian. The true static solution $R_{o}$ includes a $\xi$-dependent part $\hbar \chi_{o}$ and satisfies

$$
V\left(R_{o}(\sigma), \sigma,\right)=E_{o}
$$

Substituting $R_{o}=r_{o}+\hbar \chi_{o}$ and $V=V_{0}+\hbar \hat{V}$ in the above and expanding to first order in $\hbar$ we obtain

$$
\chi_{o}=-\frac{\hat{V}}{u_{o}}, \quad \text { where } \quad u_{o}=\left(\frac{\partial V_{0}}{\partial \phi^{D}}\right)_{o}
$$

Nevertheless, we may expand our droplet around the reference configuration $r_{o}$. Such a perturbation can be written as

$$
R=r_{o}+\hbar \chi
$$

where $\chi(\sigma)$ is a matrix and we have explicitly indicated that this is an order $\hbar$ perturbation. Correspondingly, the Poisson brackets (33) or (38) and hamiltonian (42) have to be expanded to that order.

\subsubsection{Leading-order Poisson brackets and hamiltonian}

For the Poisson brackets, the leading part of the first two terms in (38) (affine and proportional to $d^{A B C}$ ) is of order $\hbar^{0}$ and involves the scalar equilibrium term $r_{o}$ alone. In the last term the scalar part $r_{o}$ (proportional to the identity matrix $\delta^{A 0}$ ) drops out; due to the explicit $1 / \hbar$ in its coefficient, however, the $\chi$ part survives. Overall we have

$$
\left\{\chi^{A}\left(\sigma_{1}\right), \chi^{B}\left(\sigma_{2}\right)\right\}=\frac{\sqrt{\theta_{o+}}}{\hbar^{2} \rho_{o}}\left[\left(\theta_{o+}^{D \alpha}-\theta_{o+}^{\beta \alpha} \partial_{\beta} r_{o}\right) \partial_{\alpha} \delta\left(\sigma_{-}\right) \delta^{A B}+f^{A B C} \chi^{C} \delta\left(\sigma_{-}\right)\right]
$$

(Note that we reinstated the dependence of $\theta^{\alpha \beta}$ on $R$; the dependence on the matrix part $\chi$ that would complicate the Poisson brackets is down by $\hbar$ and can be neglected.)

The single-particle hamiltonian perturbed to order $\hbar$ around $r_{o}$ is

$$
V=E_{o}+\hbar u_{o}(\sigma) \chi(\sigma)+\hbar \hat{V}_{o}
$$


and this gives for the droplet hamiltonian

$$
H=H_{o}+\hbar \rho_{o} E_{o} \int \frac{d \sigma}{\sqrt{\theta_{o}}} \operatorname{tr} \chi+\hbar^{2} \rho_{o} \int \frac{d \sigma}{\sqrt{\theta_{o}}} \operatorname{tr}\left(\frac{1}{2} u_{o} \chi^{2}+\hat{V}_{o} \chi\right)
$$

$H_{o}$ is the energy of the unperturbed droplet; it is a constant and can be discarded. The next term, of order $\hbar$, is proportional to $E_{o}\left(N-N_{o}\right)$, where $N$ is the Casimir (39) and $N_{o}$ the value of the Casimir for the unperturbed droplet. It is therefore itself a Casimir and does not contribute to the equations of motion. It can be set to zero as an initial condition, corresponding to a constant-volume perturbation of the droplet (total number of particles $N$ constant). We end up with a hamiltonian of order $\hbar^{2}$ with a linear and a quadratic term in $\chi$ :

$$
H=\hbar^{2} \rho_{o} \int \frac{d \sigma}{\sqrt{\theta_{o}}} \operatorname{tr}\left(\frac{1}{2} u_{o} \chi^{2}+\hat{V}_{o} \chi\right)
$$

Finally, the equation of motion as obtained by the above hamiltonian and Poisson brackets, or from the full equation of motion (44) to first order in $\hbar$, becomes

$$
\dot{\chi}=\left(\theta_{o}^{D \alpha}-\theta_{o}^{\beta \alpha} \partial_{\beta} r_{o}\right) \partial_{\alpha}\left(u_{o} \chi+\hat{V}_{o}\right)+i\left[\chi, \hat{V}_{o}\right]
$$

Note that the factors $\hbar^{2} \rho_{o}$ have cancelled.

\subsubsection{Wess-Zumino-Witten action}

The form of the above equation of motion is suggestive. Let us define the differential operator

$$
\partial_{\tau}=\left(\theta_{o}^{D \alpha}-\theta_{o}^{\beta \alpha} \partial_{\beta} r_{o}\right) \partial_{\alpha}
$$

representing a vector field along the classical trajectory of a particle on the boundary of the droplet. Note that $\partial_{\tau}$ is properly anti-self-adjoint on the boundary of the droplet under the integration measure $d \sigma / \sqrt{\theta_{o}}$ due to the relation

$$
\partial_{\alpha}\left(\frac{\theta^{\mu \alpha}}{\sqrt{\theta_{o}}}\right)=0
$$

In terms of $\partial_{\tau}$ the equation of motion becomes

$$
\dot{\chi}+i\left[\hat{V}_{o}, \chi\right]-\partial_{\tau}\left(u_{o} \chi\right)=\partial_{\tau} \hat{V}_{o}
$$

Similarly, the Poisson brackets of $\chi$ become

$$
\left\{\chi^{A}\left(\sigma_{1}\right), \chi^{B}\left(\sigma_{2}\right)\right\}=\frac{\sqrt{\theta_{o+}}}{\hbar^{2} \rho_{o}}\left[\partial_{\tau+} \delta\left(\sigma_{-}\right) \delta^{A B}+f^{A B C} \chi^{C} \delta\left(\sigma_{-}\right)\right]
$$


We recognize the above Poisson structure as the Kac-Moody algebra of a chiral current $J_{+}=\chi$. This algebra is realized by one of the light-cone components of the current in a Wess-Zumino-Witten (WZW) model, with the corresponding light-cone coordinate $x^{+}$identified as the trajectory along which $\partial_{\tau}$ acts and the other lightcone coordinate $x^{-}$identified as time. The remaining directions of $\sigma$ appear simply as parameters.

This immediately provides a lagrangian realization of the above hamiltonian structure, in terms of the WZW action of a unitary $n \times n$ matrix field $U$. The chiral field $\chi$ is identified as the current

$$
\chi=-i U^{-1} \partial_{\tau} U
$$

Interestingly, this form for $\chi$ implies that the deformation of the droplet is generated by the unitary field $U$, in analogy with the spinless case. Specifically, consider a small deformation of the coordinates $\phi, \xi$ generated by an order- $\hbar$ generating function $\hbar \Phi(\sigma, \xi)$ :

$$
r_{o} \rightarrow r_{o}+\hbar \theta_{o}^{D \alpha} \partial_{\alpha} \Phi, \quad \sigma^{\alpha} \rightarrow \sigma^{\alpha}+\hbar \theta_{o}^{\alpha \beta} \partial_{\beta} \Phi, \quad \xi^{i} \rightarrow \xi^{i}+\hbar \theta^{i j} \partial_{j} \Phi
$$

The deformations of $\phi^{D}=r_{o}$ and $\sigma^{\alpha}$ are infinitesimal. The deformation of $\xi^{i}$, however, is of order $\hbar^{0}$ and of the same order as $\xi^{i}$; it cannot be written in the above infinitesimal form. Instead, we must write the analog of a finite canonical transformation on the spin space, which is a unitary transformation.

The action that reproduces the Poisson brackets of $\chi$ is the WZW action at critical coupling. The WZW model gives equation of motion $\partial_{-} J_{+}=0$, corresponding to $\dot{\chi}=0$, which implies a vanishing hamiltonian. The full action, then, is the WZW action minus the hamiltonian

$$
S=S_{W Z}+\hbar^{2} \rho_{o} \int d t \frac{d \sigma}{\sqrt{\theta_{o}}} \operatorname{tr}\left[-\frac{1}{2} U^{-1} \partial_{t} U U^{-1} \partial_{\tau} U+\frac{1}{2} u_{o}\left(U^{-1} \partial_{\tau} U\right)^{2}+i \hat{V}_{o} U^{-1} \partial_{\tau} U\right]
$$

The Wess-Zumino action $S_{W Z}$ can be written as an integral over a $D$-dimensional manifold whose boundary is the boundary of the droplet. Introducing a variable $s \in[0,1]$ we can take $U(s, \sigma, t)$ to be any extension of $U$ away from the boundary such that

$$
U(1, \sigma, t)=U(\sigma, t), \quad U(0, \sigma, t)=1
$$

The WZ action with the proper normalization to reproduce (57) is

$$
S_{W Z}=\frac{\hbar^{2} \rho_{o}}{6} \int d s d t \frac{d \sigma}{\sqrt{\theta_{o}}} \operatorname{tr}\left\{U^{-1} \partial_{s} U\left[U^{-1} \partial_{\tau} U, U^{-1} \partial_{t} U\right]\right\}
$$


The above WZ action can be written in a more suggestive way by choosing the $D$-dimensional manifold of integration to be the bulk of the droplet itself, identifying $s$ with $\phi^{D}$ and allowing the integration measure $1 / \sqrt{\theta}$ to vary accordingly in the bulk of the droplet. In doing that, we have to take into account the following:

- $\partial_{\tau}=\left(\theta_{o}^{D \alpha}-\theta_{o}^{\beta \alpha} \partial_{\beta} r_{o}\right) \partial_{\alpha}$ is defined on the boundary and must be appropriately extended in the bulk

- Unlike $s, \phi^{D}$ is not constant on the boundary, but rather $\phi^{D}=r_{o}(\sigma)$

- The integrand should be a closed form so that its variation reproduce the same boundary term as (62)

These actually combine to give the WZ bulk action a simple geometrical form as the integral of a $D+1$-form, in terms of the canonical two-form $\omega=\frac{1}{2} \omega_{\mu \nu} d \phi^{\mu} d \phi^{\nu}$ and the exterior derivative $d=d t \partial_{t}+d \phi^{\mu} \partial_{\mu}$. In anticommuting form notation:

$$
S_{W Z}=\frac{\hbar^{2} \rho_{o}}{(k-1) !} \int_{D} \frac{1}{3} \omega^{k-1} \operatorname{tr}\left(U^{-1} d U\right)^{3}, \quad k=\frac{D}{2}
$$

This has the form of a Kähler-Wess-Zumino term on the bulk of the droplet with $\omega$ playing the role of the Kähler structure [12. It is obviously a closed form, since both $\omega$ and $\operatorname{tr}\left(U^{-1} d U\right)^{3}$ are closed ( $d \omega=0$ is equivalent to the Jacobi identity for $\theta)$. Its variation will give the boundary integral

$$
\delta S_{W Z}=\frac{\hbar^{2} \rho_{o}}{(k-1) !} \int_{D-1} \omega_{o}^{k-1} \operatorname{tr}\left[\left(U^{-1} d U\right)^{2} U^{-1} \delta U\right]
$$

To see that this is what we want, note that $\omega^{k}$ is a top form on the phase space and thus

$$
\begin{aligned}
\omega^{k} & =k ! \sqrt{\operatorname{det} \omega} d \phi^{1} \cdots d \phi^{D}=\frac{k !}{(2 k) !} \sqrt{\operatorname{det} \omega} \epsilon_{\mu_{1} \ldots \mu_{D}} d \phi^{\mu_{1}} \cdots d \phi^{\mu_{D}} \\
\omega^{k-1} & =\frac{(k-1) !}{2(2 k-2) !} \sqrt{\operatorname{det} \omega} \epsilon_{\mu_{1} \ldots \mu_{D}} \theta^{\mu_{1} \mu_{2}} d \phi^{\mu_{3}} \cdots d \phi^{\mu_{D}}
\end{aligned}
$$

( $\sqrt{\operatorname{det} \omega}$ is really the Pfaffian of the antisymmetric matrix $\omega_{\mu \nu}$ ). Restriction of the form $\omega^{k-1}$ on the boundary will produce a factor of $\sqrt{\operatorname{det} \omega_{o}}=1 / \sqrt{\theta_{o}}$ and will induce the substitutions

$$
d \phi^{D}=d \sigma^{\alpha} \partial_{\alpha} r_{o}, \quad d U=d t \partial_{t} U+d \sigma^{\alpha} \partial_{\alpha} U
$$

So $\left(U^{-1} d U\right)^{2}=d t d \sigma^{\alpha}\left[U^{-1} \partial_{t} U, U^{-1} \partial_{\alpha} U\right]$. Taking into account the combinatorics, the terms in (65) with $\mu_{1}=D$ or $\mu_{2}=D$ reproduce the term $\theta_{o}^{D \alpha}$ in $\partial_{\tau}$, while the terms with any of the remaining $\mu$ s equal to $D$ reproduce the term $\theta_{o}^{\beta \alpha}$ in $\partial_{\tau}$. 
Finally, we may recast the full action (60) above in a more familiar form by renaming $\hat{V}_{o}=-A_{0}$ and defining the gauged time derivative

$$
D_{0} U=\partial_{t} U-i\left[A_{0}, U\right]=\partial_{t} U+i\left[\hat{V}_{o}, U\right]
$$

The action becomes

$$
S=S_{W Z}-\frac{\hbar^{2} \rho_{o}}{2} \int \frac{d t d \sigma}{\sqrt{\theta_{o}}} \operatorname{tr}\left[U^{-1}\left(D_{0} U-u_{o} \partial_{\tau} U\right) U^{-1} \partial_{\tau} U+i\left(A_{0} U^{-1}+U^{-1} A_{0}\right) \partial_{\tau} U\right]
$$

This has the form of a gauged WZW model. The last term is the extra term needed in the action to absorb the anomaly of the Wess-Zumino term under gauge transformations $U \rightarrow W^{-1} U W$. We have recovered the action of Karabali and Nair for a temporal gauge field, generalized to an arbitrary phase space droplet and with a $\sigma$-dependent potential gradient $u_{o}$.

\subsubsection{Comments on the dynamics of the model}

The expression for $\chi=-i U^{-1} \partial_{\tau} U$ implies that the deformation of the droplet is generated by the unitary field $U$, in analogy with the spinless case. Specifically, assume a small canonical transformation of the coordinates $\phi, \xi$ induced by an order$\hbar$ generating function $\hbar \Phi(\sigma, \xi)$ :

$$
r_{o} \rightarrow r_{o}+\hbar \theta_{o}^{D \alpha} \partial_{\alpha} \Phi, \quad \sigma^{\alpha} \rightarrow \sigma^{\alpha}+\hbar \theta_{o}^{\alpha \beta} \partial_{\beta} \Phi, \quad \xi^{i} \rightarrow \xi^{i}+\hbar \theta^{i j} \partial_{j} \Phi
$$

The deformations of $\phi^{D}=r_{o}$ and $\sigma^{\alpha}$ are of order $\hbar$. The deformation of $\xi^{i}$, however, is of order $\hbar^{0}$ (since $\theta^{i j}$ is of order $\hbar^{-1}$ ) and of the same order as $\xi^{i}$; it cannot be written in the above infinitesimal form. Instead, we must write the analog of a finite canonical transformation on the spin space, which is a unitary transformation.

The dynamical meaning of the above WZW structure is as follows: The operator $\mathcal{L}=u_{o} \partial_{\tau}$ generates classical motion on the manifold $\phi^{D}=r_{o}$ under the spinless part of the single-particle hamiltonian $V_{0}$. (The variable $\tau$ represents time of flight along the classical path.) In the absence of the spin-dependent (matrix) part $\hat{V}$, particles would simply move along the flow of $\mathcal{L}$, and so would the surface of the droplet. Such motion also rescales distances away from the droplet. The rescaled deformation of the surface $u_{o} \chi$, then, would evolve as a co-moving matrix: $u_{o} \dot{\chi}=\mathcal{L}\left(u_{o} \chi\right)$. The trace (scalar) part of $\chi$ represents total particle number (charge) fluctuations, while its traceless part represents spin fluctuations of the droplet. The scalar and traceless parts actually decouple, signaling spin-charge separation in this limit. 
In the absence of $\hat{V}$ the motion of the various matrix components of $\chi$ would decouple and could be described as a collection of independent abelian chiral models. The presence of the spin part $\hat{V}$, however, causes a 'Pauli rotation' of the coordinates $\xi^{i}$ and thus couples the matrix components inducing an extra unitary transformation of the matrix $\chi$ that can be understood as gauge (spin) rotation. The gauged WZW action is the proper dynamical setting for describing such motion.

The appearance of gauge structure in the problem is somewhat surprising, since we have not introduced gauge fields or considered gauge transformations. In the next section we shall complete the picture by doing that.

\section{Introducing gauge fields}

In the analysis so far we have described spin in terms of an internal compact phase space of the particles. Its canonical structure decoupled from the one of the kinematical phase space $\left(\theta^{\alpha i}=0\right)$ and any nontrivial spin dynamics arose out of the hamiltonian.

We may further couple spin and kinematical degrees of freedom by introducing nonzero phase space structure constants between the two spaces. As we shall demonstrate, this amounts to introducing nonabelian gauge fields and endows the dynamics with a nonabelian gauge symmetry. For other examples of introducing gauge degrees of freedom in the canonical description of particles or fluids see [13], [11].

\subsection{Coupling the phase spaces}

The most convenient setting for analyzing the situation is in terms of the canonical one-form formulation of the phase space. We give below the relevant facts for our purpose.

We consider a phase space $x^{\mu}$ endowed with a canonical one-form $\mathcal{A}=\mathcal{A}_{\mu} d x^{\mu}$ and a hamiltonian $V$. (In our case, $x^{\mu}$ will comprise both $\phi^{\mu}$ and $\xi^{i}$ ). $\mathcal{A}$ and $V$ could be time-dependent. The phase space action and lagrangian are

$$
L=\mathcal{A}_{\mu} \dot{x}^{\mu}-V, \quad d S=L d t=A_{\mu} d x^{\mu}-V d t
$$

which leads to the canonical two-form $\omega=d \mathcal{A}$ inverse to the Poisson structure $\theta$ :

$$
\omega_{\mu \nu}=\partial_{\mu} \mathcal{A}_{\nu}-\partial_{\nu} \mathcal{A}_{\mu}, \quad \omega_{\mu \nu} \theta^{\nu \rho}=\delta_{\mu}^{\rho}
$$


The above action has the standard phase space invariances. The first is generated by adding to the lagrangian the total time derivative of an infinitesimal phase space function $\Phi(x ; t)$

$$
\delta_{\Phi} L=\dot{\Phi}
$$

which amounts to the abelian gauge transformation

$$
\delta_{\Phi} \mathcal{A}_{\mu}=\partial_{\mu} \Phi, \quad \delta_{\Phi} V=-\partial_{t} \Phi
$$

leaving the canonical two-form $\omega$ and Poisson structure $\theta$ invariant. The other is general coordinate invariance, generated by arbitrary infinitesimal coordinate redefinitions

$$
\delta_{\epsilon} x^{\mu}=-\epsilon^{\mu}(x ; t)
$$

which is compensated by the transformation

$$
\delta_{\epsilon} \mathcal{A}_{\mu}=\partial_{\nu} \mathcal{A}_{\mu} \epsilon^{\nu}+\mathcal{A}_{\nu} \partial_{\mu} \epsilon^{\nu}, \quad \delta_{\epsilon} V=\partial_{\mu} V \epsilon^{\mu}-\mathcal{A}_{\mu} \partial_{t} \epsilon^{\mu}
$$

(The minus sign in (74) is put to stress the fact that this is a "passive" transformation of coordinates.) The above can be rewritten as

$$
\delta_{\epsilon} \mathcal{A}_{\mu}=-\omega_{\mu \nu} \epsilon^{\nu}+\partial_{\mu}\left(\mathcal{A}_{\nu} \epsilon^{\nu}\right), \quad \delta_{\epsilon} V=\left(\partial_{t} \mathcal{A}_{\mu}+\partial_{\mu} V\right) \epsilon^{\mu}-\partial_{t}\left(\mathcal{A}_{\mu} \epsilon^{\mu}\right)
$$

involving canonically invariant quantities and an abelian gauge transformation generated by $\Phi_{\epsilon}=\mathcal{A}_{\nu} \epsilon^{\nu}$.

Canonical transformations are a special case of coordinate transformations leaving the Poisson structure invariant. Choosing as coordinate deformation parameters

$$
\epsilon_{c}^{\mu}=\left\{x^{\mu}, \Phi\right\}=\theta^{\mu \nu} \partial_{\nu} \Phi
$$

we get for the change in $\mathcal{A}_{\mu}$ and $V$

$$
\delta_{\epsilon_{c}} \mathcal{A}_{\mu}=\partial_{\mu}\left(-\Phi+\theta^{\nu \rho} \mathcal{A}_{\nu} \partial_{\rho} \Phi\right), \quad \delta_{\epsilon_{c}} V=\{V, \Phi\}+\partial_{t} \mathcal{A}_{\mu} \theta^{\mu \nu} \partial_{\nu} \Phi
$$

This corresponds to an abelian gauge transformation on the $\mathcal{A}_{\mu}$ that leaves $\theta^{\mu \nu}$ invariant. For time-independent $\mathcal{A}_{\nu}, V$ transforms by a canonical transformation, while a time dependence in $\mathcal{A}_{\mu}$ contributes an extra correction.

We specialize now to the phase space of interest $\left(\phi^{\mu}, \xi^{i}\right)$. The original lagrangian is

$$
L=\mathcal{A}_{\mu}(\phi) \dot{\phi}^{\mu}+\mathcal{A}_{i}(\xi) \dot{\xi}^{i}-V
$$


The Poisson structure decouples the spin and kinematical phase spaces, which reflects to the fact that $\mathcal{A}_{\alpha}(\phi)$ and $\mathcal{A}_{i}(\xi)$ depend only on their corresponding phase space variables, ensuring $\partial_{i} \mathcal{A}_{\alpha}=\partial_{\alpha} \mathcal{A}_{i}=0$.

We shall couple $\xi$ and $\phi$ by relaxing the above condition. In doing so we do not want to distort the structure of the internal phase space. Its volume, as well as the area of all noncontractible two-submanifolds, must remain fixed to appropriate integers for a consistent quantization (cf. to monopole quantization for $S^{2}$ and $K$ eigenvalue quantization for the grassmanian case of section 3.1). It should also stay a homogeneous space to allow for a linear Poisson algebra in terms of appropriate spin generators. We shall, therefore, keep its one-form $\mathcal{A}_{i}(\xi)$ the same as above and independent of $\phi^{i}$ and shall write it $\hbar \bar{A}_{i}$ to explicitly indicate the fact that it is of order $\hbar$.

We will however allow $\mathcal{A}_{\alpha}$ to depend on $\xi$. The new one-form will consist of the old one, denoted by $\bar{A}_{\alpha}$, plus a $\xi$-dependent order- $\hbar$ perturbation $\hbar A_{i}$. We also write the hamiltonian in the form $\bar{V}+\hbar \hat{V}=\bar{V}-\hbar A_{0}$, taking a hint from the previous section in renaming $\hat{V}$ to $-A_{0}$. Further, we will allow $A_{\alpha}$ and $A_{0}$ to be time-dependent. Altogether the lagrangian becomes

$$
L=\bar{A}_{\alpha}(\phi) \dot{\phi}^{\alpha}+\hbar \bar{A}_{i}(\xi) \dot{\xi}^{i}-\bar{V}(\phi)+\hbar A_{\alpha}(\phi, \xi, t) \dot{\phi}^{\alpha}+\hbar A_{0}(\phi, \xi, t)
$$

In terms of scales, $\omega_{i j}=\hbar\left(\partial_{i} \bar{A}_{j}-\partial_{j} \bar{A}_{i}\right)$ is of order $\hbar$ and $\theta^{i j}=\left(\omega^{-1}\right)^{i j}$ is of order $1 / \hbar$ as it should.

\subsection{Gauge transformations}

We come now to the issue of gauge transformations in the spin space of our phase space structure (80). Interpreting $\xi$ as spin variables, we understand that gauge transformations should amount to local rotations of the $\xi$-coordinates in their phase space; that is, canonical transformations in the $\xi$ space that depend on the kinematical phase space coordinates. These will be generated by an order- $\hbar$ function $\hbar \Phi(\phi, \xi, t)$

$$
\delta \xi^{i}=-\hbar\left\{\xi^{i}, \Phi\right\}_{\xi}=-\hbar \theta^{i j} \partial_{j} \Phi, \quad \delta \phi^{\alpha}=0
$$

Note that the above is not a canonical transformation on the full space, since the kinematical coordinates $\phi$ are not transformed and the Poisson bracket in the transformation of $\xi$ is restricted. The canonical one-form and hamiltonian are transformed according to (76), which implies for $A_{\alpha}, A_{0}$ and $\tilde{A}_{i}$ :

$$
\delta A_{\alpha}=\hbar \partial_{i} A_{\alpha} \theta^{i j} \partial_{j} \Phi+\hbar \partial_{\alpha}\left(\theta^{i j} A_{i} \partial_{j} \Phi\right)
$$




$$
\begin{aligned}
\delta A_{0} & =\hbar \partial_{i} A_{0} \theta^{i j} \partial_{j} \Phi+\hbar \partial_{0}\left(\theta^{i j} A_{i} \partial_{j} \Phi\right) \\
\delta \bar{A}_{i} & =-\partial_{i} \Phi+\hbar \partial_{i}\left(\theta^{i j} \bar{A}_{i} \partial_{j} \Phi\right)
\end{aligned}
$$

We observe that $A_{\alpha}$ and $A_{0}$ transform as the space and time components of a one-form. Calling $x^{0}=t$ and using middle-greek letters for spacetime indices, $\mu, \nu=0,1, \ldots D$ (not to be confused with $\mu, \nu=1,2, \ldots D$ used in early sections), we can write the above as

$$
\begin{aligned}
\delta A_{\mu} & =\hbar\left\{A_{\mu}, \Phi\right\}_{\xi}+\partial_{\mu}\left(\hbar \theta^{i j} \bar{A}_{i} \partial_{j} \Phi\right) \\
\delta \bar{A}_{i} & =\partial_{i}\left(-\Phi+\hbar \theta^{i j} \bar{A}_{i} \partial_{j} \Phi\right)
\end{aligned}
$$

Under the above transformation, the spin one-form transforms away from its reference value $\bar{A}_{i}$ by a total derivative. We may restore it to its original form by adding to the lagrangian the total derivative of $\Phi-\hbar \theta^{i j} \bar{A}_{i} \partial_{j} \Phi$. This further transforms $A_{\mu}$ to

$$
\delta_{g} A_{\mu}=\partial_{\mu} \Phi+\hbar\left\{A_{\mu}, \Phi\right\}_{\xi}
$$

This has exactly the form of a nonabelian gauge transformation. Indeed, upon quantization of the spin space, $\xi$-dependent quantities such as $\Phi$ and $A_{\alpha}$ become matrices and Poisson brackets become $(1 / i \hbar)$ commutators. So the above transform becomes

$$
\begin{aligned}
\delta_{g} A_{\mu} & =\partial_{\mu} \Phi-i\left[A_{\mu}, \Phi\right] \\
\delta \xi^{i} & =i\left[\xi^{i}, \Phi\right]
\end{aligned}
$$

which corresponds to the transformation of a covariant derivative $D_{\mu}=\partial_{\mu}-i A_{\mu}$ and an anti-covariant spin matrix $\xi^{i}$ under an infinitesimal unitary rotation $U=1+i \Phi$ :

$$
\begin{aligned}
D_{\mu} & \rightarrow U D_{\mu} U^{-1} \\
\xi^{i} & \rightarrow U^{-1} \xi^{i} U
\end{aligned}
$$

The $A_{\mu}$, thus, can be rightfully considered as nonabelian gauge fields on the phase space. Gauge transformations are local rotations of the spin coordinates, which is a passive transformation, reflected in the anti-covariant nature of $\xi^{i}$. We recover the dynamics of a single spinning (in fact, colored) particle interacting with a nonabelian gauge field in phase space.

The group of the nonabelian gauge transformations is determined by the realization of the spin phase space. Gauge invariance derives from canonical spin transformations and therefore inherits the full $S U(n)$ symmetry group of the spin 
Hilbert space. Its realization, however, may be restricted by the physics of the problem (cf. the discussion of section 3.2.2).

As an example, in the realization in terms of a spin- $\frac{n-1}{2}$ representation of $S U(2)$, as argued in section 3.2.2, only operators linear in the spin variables $S^{i}=\hbar \xi^{i}$ are natural and thus the expression for $A_{\mu}$ will be restricted to

$$
A_{\mu}(\phi, \xi, t)=A_{\mu}^{i}(\phi, t) S^{i}, \quad i=1,2,3
$$

A general $S U(n)$ transformation will take the above $A_{\mu}$ away from this form. Only unitary transformations in the $S U(2)$ subgroup transforming linearly the $\xi^{i}$ will be proper gauge transformations, the field $A_{\mu}$ being in the spin- $\frac{n-1}{2}$ representation of the group. The grassmanian representation with a Kirillov form $K=\operatorname{diag}\left(n_{1}, \ldots n_{M}\right)$ and a corresponding linear restriction for the form of $A_{\mu}$ would produce an $S U(M)$ gauge group in the representation corresponding to Young tableau with $n_{i}$ blocks in row $i$.

\subsection{Equations of motion}

The above interpretation can be further justified by looking at the single-particle equations of motion in the presence of the extra coupling between $\phi$ and $\xi$ due to the (time-dependent) $A_{\alpha}$ and $A_{0}$. These can be obtained by varying the action with an arbitrary $\epsilon^{\mu}$ and setting the variation to zero. Using (76) this implies

$$
\omega_{\mu \nu} \dot{x}^{\nu}=0
$$

where $x^{\mu}$ stands for $\left\{x^{0}, \phi^{\alpha}, \xi^{i}\right\}$. (In principle, since we do not vary $x^{0}=t$, we only obtain the above equations for $\mu \neq 0$. The $\mu=0$ equation, however, holds true as a corollary of the remaining equations, due to the identity $\omega_{\mu \nu} \dot{x}^{\mu} \dot{x}^{\nu}=0$, so we obtain the full covariant set of equations.) These can also be written as

$$
\dot{x}^{\alpha}=\theta^{\alpha \beta}\left(\partial_{\beta} V+\partial_{0} A_{\beta}\right)=\left\{x^{\alpha}, V\right\}_{s p}+\theta^{\alpha \beta} \partial_{0} A_{\beta} \quad(\alpha, \beta \neq 0)
$$

which, for time-independent $A_{\alpha}$, reduce to the usual canonical equations of motion. Applying the above equation (88) to the case of the lagrangian (80) for $\phi^{\alpha}, \xi^{i}$ we obtain

$$
\begin{aligned}
{\left[\bar{\omega}_{\alpha \beta}+\hbar\left(\partial_{\alpha} A_{\beta}-\partial_{\beta} A_{\alpha}\right)\right] \dot{\phi}^{\beta} } & =\partial_{\alpha} \bar{V}+\hbar\left(\partial_{0} A_{\alpha}-\partial_{\alpha} A_{0}+\hbar \partial_{i} A_{\alpha} \dot{\xi}^{i}\right) \\
\bar{\omega}_{i j} \dot{\xi}^{j}+\hbar\left(\partial_{i} A_{0}+\partial_{i} A_{\alpha} \dot{\phi}^{\alpha}\right) & =0
\end{aligned}
$$


where $\bar{\omega}=d \overline{\mathcal{A}}$ is the reference (uncoupled) canonical two-form. The above equations can be combined and rewritten as

$$
\begin{aligned}
& \left(\bar{\omega}_{\alpha \beta}+\hbar F_{\alpha \beta}\right) \dot{\phi}^{\beta}=\partial_{\alpha} \bar{V}+\hbar F_{0 \alpha} \\
& \dot{\xi}^{i}-\hbar\left\{A_{0}+\dot{\phi}^{\alpha} A_{\alpha}, \xi^{i}\right\}_{\xi}=0
\end{aligned}
$$

where

$$
F_{\mu \nu}=\partial_{\mu} A_{\nu}-\partial_{\nu} A_{\mu}+\hbar\left\{A_{\mu}, A_{\nu}\right\}_{\xi}
$$

is the nonabelian field strength of the gauge field $A_{\mu}$. These equations have the structure of the equations of motion of a particle with nonabelian degrees of freedom and only involve gauge covariant quantities: the first is the standard 'minimal' coupling of the particle's coordinates to a field strength coupled to its (nonabelian) charge (with a scalar potential $\bar{V}$ and an electric field $F_{0 \alpha}$ ), while the second is the covariant parallel transport of the spin over the particle's phase-space-time trajectory. Due to the anti-covariant (passive) nature of $\xi$, its equation of motion involves covariant derivatives with the opposite sign for $A_{\mu}$. Observables of the form

$$
Q=\int d \xi Q_{i} \xi^{i} \rightarrow \operatorname{tr}\left(Q_{i} \xi^{i}\right)
$$

are gauge invariant, while $Q_{i}$ transform covariantly.

\subsection{Gauged droplet dynamics}

The generalization of the droplet construction for the gauged phase space considered above is straightforward. The Poisson structure is, now, time-dependent, involving the gauge fields $A_{\alpha}$, but the counting of states and fermion exclusion principle that led to constant-density droplets remain the same. The construction of the boundary field Poisson brackets, hamiltonian and equation of motion for the classical theory is

as in section 3.2 with the generalized form of $\theta_{b}^{\alpha \beta}$, involving gauge fields, appearing in the formulae.

The quantization of the spin space in this case presents some new ordering ambiguities, since we cannot any more assume that $\theta^{\mu \nu}$ is independent of $\phi^{D}$ and $R$. The proper ordering of the full nonlinear matrix Poisson brackets for $R^{a b}$ will be partly determined by the requirement that they satisfy the Jacobi identity.

To leading-order in $\hbar$, however, there are no ambiguities. All nonlinear terms in the Poisson structure that would require ordering are of higher order and can be ignored. The leading terms reproduce a fully gauged Kac-Moody algebra, as we shall demonstrate. 
The canonical two-form as derived from (80), denoted $\tilde{\omega}_{\mu \nu}$, consists of a leading part $\omega_{\mu \nu}$ and an order- $\hbar$ part:

$$
\begin{aligned}
\tilde{\omega}_{\alpha \beta} & =\omega_{\alpha \beta}+\hbar\left(\partial_{\alpha} A_{\beta}-\partial_{\beta} A_{\alpha}\right) \\
\tilde{\omega}_{i \alpha} & =\hbar \partial_{i} A_{\alpha} \\
\tilde{\omega}_{i j} & =\omega_{i j}=\hbar\left(\partial_{i} \bar{A}_{j}-\partial_{j} \bar{A}_{i}\right)
\end{aligned}
$$

The expansion of $\tilde{\theta}=\tilde{\omega}^{-1}$ in $\hbar$ is complicated by the fact that, to leading order, $\omega_{i j}$ vanishes and so the $\hbar^{0}$ part of $\omega_{\mu \nu}$ is singular. To overcome this, we temporarily change the scale of the spin coordinates by incorporating a factor of $\sqrt{\hbar}$ in each $\xi^{i}$, which has the effect:

$$
\omega_{i \alpha} \rightarrow \hbar^{-\frac{1}{2}} \omega_{i \alpha}, \quad \omega_{i j} \rightarrow \hbar^{-1} \omega_{i j}
$$

The rescaled $\omega$ becomes:

$$
\begin{aligned}
\tilde{\omega}_{\alpha \beta} & =\bar{\omega}_{\alpha \beta}+\hbar\left(\partial_{\alpha} A_{\beta}-\partial_{\beta} A_{\alpha}\right) \\
\tilde{\omega}_{i \alpha} & =\hbar^{\frac{1}{2}} \partial_{i} A_{\alpha} \\
\tilde{\omega}_{i j} & =\bar{\omega}_{i j}=\partial_{i} \bar{A}_{j}-\partial_{j} \bar{A}_{i}
\end{aligned}
$$

This is an order $\hbar^{\frac{1}{2}}$ perturbation $\delta \omega$ over a nonsingular form $\bar{\omega}_{\mu \nu}$. We can calculate the inverse in the standard expansion,

$$
\tilde{\theta}=\bar{\theta}-\bar{\theta} \delta \omega \bar{\theta}+\bar{\theta} \delta \omega \bar{\theta} \delta \omega \bar{\theta}+\ldots
$$

$\left(\bar{\theta}=\bar{\omega}^{-1}\right)$. The result to order $\hbar$ is:

$$
\begin{aligned}
\tilde{\theta}^{\alpha \beta} & =\bar{\theta}^{\alpha \beta}-\hbar \bar{\theta}^{\alpha \gamma} F_{\gamma \delta} \bar{\theta}^{\delta \beta} \\
\tilde{\theta}^{\alpha i} & =\hbar^{\frac{1}{2}} \bar{\theta}^{\alpha \beta} \bar{\theta}^{j i} \partial_{j} A_{\beta} \\
\tilde{\theta}^{i j} & =\bar{\theta}^{i j}+\hbar \bar{\theta}^{i k} \bar{\theta}^{j l} \bar{\theta}^{\alpha \beta} \partial_{k} A_{\alpha} \partial_{l} A_{\beta}
\end{aligned}
$$

We see that we now have a nonvanishing $\theta^{\alpha i}$. Finally, we may restore the original scale of the spin coordinates, which amounts to $\tilde{\theta}^{\alpha i} \rightarrow \hbar^{-\frac{1}{2}} \tilde{\theta}^{\alpha i}, \tilde{\theta}^{i j} \rightarrow \hbar^{-1} \tilde{\theta}^{i j}$. We also revert to the original spin Poisson structure, $\theta^{\alpha \beta}=\bar{\theta}^{\alpha \beta}, \theta^{i j}=\hbar^{-1} \bar{\theta}^{i j}$. The final result is

$$
\begin{aligned}
\tilde{\theta}^{\alpha \beta} & =\theta^{\alpha \beta}-\hbar \theta^{\alpha \gamma} F_{\gamma \delta} \theta^{\delta \beta} \\
\tilde{\theta}^{\alpha i} & =\hbar \theta^{\alpha \beta} \theta^{j i} \partial_{j} A_{\beta} \\
\tilde{\theta}^{i j} & =\theta^{i j}+\hbar^{2} \theta^{i k} \theta^{j l} \theta^{\alpha \beta} \partial_{k} A_{\alpha} \partial_{l} A_{\beta}
\end{aligned}
$$

Similarly, the determinant $\operatorname{det} \theta=(\operatorname{det} \omega)^{-1}$ will receive corrections according to

$$
\operatorname{det} \omega=\operatorname{det} \bar{\omega}\left[1+\operatorname{tr}(\bar{\theta} \delta \omega)-\frac{1}{2} \operatorname{tr}(\bar{\theta} \delta \omega)^{2}+\frac{1}{2}[\operatorname{tr}(\bar{\theta} \delta \omega)]^{2}+\ldots\right]
$$


and these will be of higher order in $\hbar$.

We may now use the new expressions (99) in the Poisson brackets for the boundary field (22). The new terms $\tilde{\theta}^{\alpha i}$ appear with derivatives acting on $R$ or $\delta$. Such terms, acting on a function $g(=R$ or $\delta)$ create new terms in the Poisson brackets of the form

$$
\theta^{\alpha i} \partial_{i} g=\hbar \theta^{\alpha \beta} \theta^{j i} \partial_{j} A_{\beta} \partial_{i} g=\hbar \theta^{\alpha \beta}\left\{A_{\beta}, g\right\}_{\xi}
$$

Upon quantization of the spin space, $\hbar\left\{A_{\beta}, g\right\} \rightarrow-i\left[A_{\beta}, g\right]$ and the above terms become commutators. Combined with the corresponding term $\theta^{\alpha \beta} \partial_{\beta}$ they give

$$
\theta^{\alpha \beta} \partial_{\beta} g+\theta^{\alpha i} \partial_{i} g=\theta^{\alpha \beta}\left(\partial_{\beta} g-i\left[A_{\beta}, g\right]\right)=\theta^{\alpha \beta} D_{\beta} g
$$

Their net effect is to gauge all the derivatives appearing in the Poisson brackets. This is the leading change in $\hbar$. Other terms will produce higher order effects. For instance, the new term in $\tilde{\theta}^{i j}$ will produce the term

$$
\theta^{\alpha \beta}\left[R, A_{\alpha}\right]\left[A_{\beta}, \delta\right]
$$

Although this does not involve explicit factors of $\hbar$, upon putting $R=r_{o}+\hbar \chi$ the contribution of the scalar leading term $R_{o}$ vanishes and the above term is of order $\hbar$.

Altogether and obtain

$$
\left\{\chi^{A}\left(\sigma_{1}\right), \chi^{B}\left(\sigma_{2}\right)\right\}=\frac{\sqrt{\theta_{o+}}}{\hbar^{2} \rho_{o}}\left[\left(\theta_{o+}^{0 \alpha}-\theta_{o+}^{\beta \alpha} \partial_{\beta} r_{o}\right) D_{\alpha}^{A B} \delta\left(\sigma_{-}\right)+f^{A B C} \chi^{C} \delta\left(\sigma_{-}\right)\right]
$$

where $D_{\alpha}^{A B}$ is the adjoint expression of the covariant derivative $D_{\alpha}$

$$
D_{\alpha}^{A B}=\delta^{A B} \partial_{\alpha}-f^{A B C} A_{\alpha}^{C}
$$

and $D_{\beta} r_{o}=\partial_{\beta} r_{o}$ since $r_{o}$ is a scalar. Similarly, the hamiltonian obtains as

$$
H=\hbar^{2} \rho_{o} \int \frac{d \sigma}{\sqrt{\theta_{o}}} \operatorname{tr}\left(\frac{1}{2} u_{o} \chi^{2}-A_{0} \chi\right)
$$

We may define as before a derivative along the direction of classical motion on the boundary and the corresponding covariant version:

$$
D_{\tau}=\left(\theta_{o}^{D \alpha}-\theta_{o}^{\beta \alpha} \partial_{\beta} r_{o}\right) D_{\alpha}
$$

In terms of $D_{\tau}$ the equation of motion becomes

$$
D_{0} \chi-D_{\tau}\left(u_{o} \chi\right)=F_{0 \tau}
$$


where

$$
F_{0 \tau}=\left[D_{0}, D_{\tau}\right]
$$

Similarly, the Poisson brackets of $\chi$ become

$$
\left\{\chi^{A}\left(\sigma_{1}\right), \chi^{B}\left(\sigma_{2}\right)\right\}=\frac{\sqrt{\theta_{o+}}}{\hbar^{2} \rho_{o}}\left[D_{\tau}^{A B}\left(\sigma_{-}\right) \delta\left(\sigma_{-}\right)+f^{A B C} \chi^{C} \delta\left(\sigma_{-}\right)\right]
$$

We obtain a gauged Kac-Moody algebra and corresponding equation of motion for the chiral current $\chi=J_{+}$. As before, this structure derives from the action of a fully gauged Wess-Zumino-Witten model in the space defined by classical motion trajectories and time, integrated over the remaining phase space variables. In terms of a unitary field $U$ we have

$$
\chi=-i U^{-1} D_{\tau} U
$$

and the action is

$$
\begin{aligned}
S & =\hbar^{2} \rho_{o} \int \frac{d t d \sigma}{\sqrt{\theta_{o}}} \operatorname{tr}\left[-\frac{1}{2} U^{-1}\left(D_{0}-u_{o} D_{\tau}\right) U U^{-1} D_{\tau} U\right] \\
& +\frac{\hbar^{2} \rho_{o}}{(k-1) !} \int_{D-1} \omega_{o}^{k-1} \operatorname{tr}\left(A U^{-1} d U+A d U U^{-1}+A U^{-1} A U\right) \\
& +\frac{\hbar^{2} \rho_{o}}{(k-1) !} \int_{D} \frac{1}{3} \omega^{k-1} \operatorname{tr}\left(U^{-1} d U\right)^{3}
\end{aligned}
$$

with $D=2 k$. The first term is the gauged kinetic term on the boundary. The last term is the standard Wess-Zumino term; it does not involve gauge fields and is obtained by integrating the Wess-Zumino form over the bulk of the droplet with an appropriately extended unitary field $U$ as in section 3.3. The middle term is defined on the boundary of the droplet and involves the gauge fields $A_{0}$ and $A_{\tau}$; it is needed to absorb the gauge non-invariance of the Wess-Zumino term and contributes the term $F_{0 \tau}$ in the equation of motion for $\chi(108)$.

Overall, we have recovered the action of [10] for a fully general gauge field, generalized to an arbitrary phase space droplet and with a $\sigma$-dependent potential gradient $u_{o}$.

\section{Conclusions and discussion}

We have presented an analysis of the phase space dynamics of droplets representing fermions with internal degrees of freedom in an arbitrary phase space and derived their hamiltonian and canonical structure. 
To leading order in $\hbar$ we recovered the WZW chiral action of edge excitations. In the nonlinear theory we do not have an explicit form for the action. This is not crucial, since we have derived the complete hamiltonian dynamics, but it remains an issue for further investigation, especially if we are interested in applying pathintegral or effective field theory techniques.

The nature of the obtained theories is halfway between classical and quantum: spin is quantized and gives rise to a matrix structure, while phase space coordinates are still treated classically. As such, it is reminiscent of the matrix formulation of the quantum Hall effect [14]. The exact correspondence between the two formulations, if any, should be further examined.

Finally, the theories derived in this paper represent a nonabelian phase space bosonization of the fermionic systems they describe. Just as in the abelian case, however, this bosonization fails quantum mechanically in dimensions higher than $D=2$. The main problems are, first, that this theory overestimates the degrees of freedom of the system, due to the infinity of excitations normal to the direction of propagation and, second, that the theory is essentially local in phase space and thus does not take into account processes where fermions would undergo transitions to faraway phase space states. This is an issue that will be treated in an upcoming publication.

Acknowledgements: I would like to thank D. Karabali and V.P. Nair for useful comments on the manuscript. This research was supported in part by the National Science Foundation under grant PHY-0353301 and by the CUNY Research Foundation under grant PSC-CUNY-66565-0035.

\section{References}

[1] S. R. Coleman, "Quantum Sine-Gordon Equation As The Massive Thirring Model," Phys. Rev. D 11, 2088 (1975); S. Mandelstam, "Soliton Operators For The Quantized Sine-Gordon Equation," Phys. Rept. 23 (1976) 307.

[2] A. Jevicki and B. Sakita, "The Quantum Collective Field Method And Its Application To The Planar Limit," Nucl. Phys. B 165, 511 (1980); S. R. Das and A. Jevicki, "String Field Theory And Physical Interpretation Of D $=1$ Strings," Mod. Phys. Lett. A 5, 1639 (1990). 
[3] E. Witten, "Nonabelian Bosonization In Two Dimensions," Commun. Math. Phys. 92, 455 (1984).

[4] G. W. Semenoff, "Canonical Quantum Field Theory With Exotic Statistics," Phys. Rev. Lett. 61, 517 (1988); G. W. Semenoff and P. Sodano, "Exotic Spin And Statistics In (2+1)-Dimensional Canonical Quantum Field Theory," Nucl. Phys. B 328, 753 (1989); T. Matsuyama, "Canonical Structures And BoseFermi Transmutations In (2+1)-Dimensional U(1) Gauge Theories," Phys. Lett. B 228, 99 (1989).

[5] J. Polchinski, "Classical limit of (1+1)-dimensional string theory," Nucl. Phys. B 362, 125 (1991).

[6] A. P. Polychronakos, "Chiral actions from phase space (quantum Hall) droplets," arXiv:hep-th/0408194.

[7] D. Karabali and V. P. Nair, "Quantum Hall effect in higher dimensions," Nucl. Phys. B 641, 533 (2002) arXiv:hep-th/0203264; "The effective action for edge states in higher dimensional quantum Hall systems," Nucl. Phys. B 679, 427 (2004) arXiv:hep-th/0307281.

[8] S. C. Zhang and J. p. Hu, "A Four Dimensional Generalization of the Quantum Hall Effect," Science 294, 823 (2001) arXiv:cond-mat/0110572; Phys. Rev. B 41, 12838 (1990) arXiv:cond-mat/0112432; B. A. Bernevig, C. H. Chern, J. P. Hu, N. Toumbas and S. C. Zhang, "Effective field theory description of the higher dimensional quantum Hall liquid," Annals Phys. 300, 185 (2002) arXiv:cond-mat/0206164.

[9] B. Sakita, "Collective variables of fermions and bosonization," Phys. Lett. B 387, 118 (1996) arXiv:hep-th/9607047; R. Ray and B. Sakita, "Bulk and edge excitations of a $\nu=1$ Hall ferromagnet," arXiv:cond-mat/0105626.

[10] D. Karabali and V. P. Nair, "Edge states for quantum Hall droplets in higher dimensions and a generalized WZW model," arXiv:hep-th/0403111.

[11] R. Jackiw, V. P. Nair, S. Y. Pi and A. P. Polychronakos, "Perfect fluid theory and its extensions," J. Phys. A 37, R327 (2004) arXiv:hep-ph/0407101.

[12] V. P. Nair and J. Schiff, "A Kahler-Chern-Simons Theory And Quantization Of Instanton Moduli Spaces," Phys. Lett. B 246, 423 (1990); "Kahler Chern- 
Simons theory and symmetries of antiselfdual gauge fields," Nucl. Phys. B 371, 329 (1992).

[13] A.P. Balachandran, G. Marmo, B-S. Skagerstam and A. Stern, Gauge Symmetries and Fibre Bundles, Springer-Verlag (1983); C. h. Chou, V. P. Nair and A. P. Polychronakos, "On the electromagnetic interactions of anyons," Phys. Lett. B 304, 105 (1993) arXiv:hep-th/9301037; B. Bistrovic, R. Jackiw, H. Li, V. P. Nair and S. Y. Pi, "Non-Abelian fluid dynamics in Lagrangian formulation," Phys. Rev. D 67, 025013 (2003) arXiv:hep-th/0210143.

[14] L. Susskind, "The quantum Hall fluid and non-commutative Chern Simons theory," arXiv:hep-th/0101029 A. P. Polychronakos, "Quantum Hall states as matrix Chern-Simons theory," JHEP 0104, 011 (2001) arXiv:hep-th/0103013; "Quantum Hall states on the cylinder as unitary matrix Chern-Simons theory," JHEP 0106, 070 (2001) arXiv:hep-th/0106011. 